\title{
Vol. 66, No. 1
}

In the report "Prevalence of Perceived Food and Housing Security - 15 States, 2013," on page 15, the website in reference 3 should be https://www.surgeongeneral.gov/priorities/ prevention/2012-npc-action-plan.pdf. 